\title{
Digitalisierung als Grundlage wertvoller Zusammenarbeit - Die Gestaltung von Service-Ökosystemen in den personennahen Dienstleistungen
}

\author{
Simon Fischer (D) - Anna Lux (D) - Ricardo Guerrero $(\mathbb{D})$ \\ Rangina Ahmad $(\mathbb{D} \cdot$ Lisa Lohrenz $(\mathbb{D}) \cdot$ Christoph Lattemann $(\mathbb{D})$
}

Eingegangen: 31. März 2020 / Angenommen: 22. Juni 2020 / Online publiziert: 6. Juli 2020

(C) Der/die Autor(en) 2020

Zusammenfassung Die Erwartungen von Kunden an Sach- und Dienstleistungen haben sich verändert. Einerseits stellen sie höhere Anforderungen in Bezug auf Qualität, Komfort und Personalisierung. Andererseits möchten sie gestaltend zur Erreichung dieser Werte beitragen. Um weiterhin am Markt konkurrieren zu können, müssen Unternehmen traditionelle Kunden- und Anbieterrollen ebenso hinter sich lassen, wie die Vorstellung, dass sie im Alleingang Innovationen schaffen zu können. Service-Ökosysteme werden in diesem Kontext zum Schlüssel innovativer Wertangebote und führen aktuelle Trends der Dienstleistungsentwicklung zusammen. In Symbiose mit digitalen Technologien werden sie zu einem attraktiven Instrument für die Schaffung von losen, heterogenen Partnernetzwerken, die gemeinsam

\footnotetext{
S. Fischer $(\bowtie) \cdot$ R. Guerrero $\cdot$ C. Lattemann Jacobs University Bremen, Bremen, Deutschland

E-Mail: si.fischer@jacobs-university.de

R. Guerrero

E-Mail: r.guerrero@jacobs-university.de

C. Lattemann

E-Mail: c.lattemann@jacobs-university.de

A. Lux · R. Ahmad · L. Lohrenz

Technische Universität Braunschweig, Braunschweig, Deutschland

A. Lux

E-Mail: anna.lux@tu-braunschweig.de

R. Ahmad

E-Mail: rangina.ahmad@tu-braunschweig.de

L. Lohrenz

E-Mail: 1.lohrenz@tu-braunschweig.de

C. Lattemann

University of Agder, Kristiansand, Norwegen
} 
innovative Dienstleistungen entwickeln und anbieten. Anhand von acht Fallbeispielen zeigt der vorliegende Beitrag auf, wie auch Unternehmen aus dem Bereich der personennahen Dienstleistungen dieses Instrument zur Aktivierung des eigenen Digitalisierungspotenzials heranziehen können. Einen Rahmen zur vergleichenden Analyse und Gestaltung kann hierbei die ,Service Canvas', der vom Bundesministerium für Bildung und Forschung (BMBF) geförderten Begleitforschung BeDien, bieten. Betrachtungspunkte wie Individualisierung, Integration, Kollaboration und Digitale Services decken Stärken, Schwerpunkte und Entwicklungspotenziale von Service-Ökosystemen auf und geben so Anhaltspunkte, wie sich Digitalisierung als Grundlage für die Zusammenarbeit zielgerichtet einsetzen lässt.

Schlüsselwörter Service-Ökosystem · Personennahe Dienstleistungen ·

Digitalisierung $\cdot$ Individualisierung $\cdot$ Kollaboration $\cdot$ Fallstudie

\section{Digitalization as a Basis for Valuable Collaboration-The Design of Service-Ecosystems for Personal Services}

Abstract Customers' expectations towards goods and services have changed. On the one hand, customers place higher demands on quality, comfort and personalization. On the other hand, they want to be taken into consideration to contribute to this value creation. In order to continue to compete in the market, companies must leave traditional customer and supplier roles aside as well as the idea of being able to create innovations on their own. Service ecosystems become the key to develop innovative value propositions and bring together current trends in service development. In symbiosis with digital technologies, they become an attractive tool for creating loose, heterogeneous partner networks. Based on eight case studies, this article reveals how companies in the field of human-centered services, or the so-called 'personal services', use their service ecosystems to achieve their own digitalization potential. A framework for comparative analysis and design can be provided by the 'Service Canvas' of the BMBF-funded research project BeDien. Different elements such as individualization, integration, collaboration and digital services reveal the strengths, advantages and development potential of service ecosystems and thus, provide indications on how digitalization can be used in a systematic manner as a basis for collaboration.

Keywords Service ecosystem · Human-centered services · Digitalization · Individualization · Collaboration · Case study

\section{Motivation und Zielsetzung}

Partnerschaftliche, netzartige und vertrauensvolle Beziehungsgeflechte bilden heutzutage die Grundlage für erfolgreiche Angebote. Längst geht es nicht mehr darum ein einzelnes Produkt anzubieten, sondern marktfähige Komplettlösungen aufzubauen. Um zukunftsfähig zu bleiben und den steigenden Ansprüchen von Kunden hinsichtlich Qualität, Komfort und Personalisierung von Produkten und Dienstleistungen 
gerecht zu werden, müssen Unternehmen eine vernetzte Wertschöpfung anstreben. Die eigenen Kompetenzen durch Zusammenarbeit mit anderen Akteuren (Kunden sowie Anbietern) zu einem möglichst vollständigen Ensemble zu ergänzen, ist der Schlüssel zu innovativen Wertangeboten. In der Dienstleistungsentwicklung lassen sich Trends zu solchen Service-Ökosystemen seit geraumer Zeit beobachten.

Durch die zunehmend populäre theoretische Perspektive der Service-dominierten Logik (SDL) (Vargo und Lusch 2004), findet bereits seit einigen Jahren ein Umdenken sowohl bei Dienstleistern als auch Produzenten in der Praxis statt. Die Fokussierung auf Gesamtlösungen für den Kunden macht deutlich, dass innovative Wertangebote im kollaborativen Zusammenschluss verschiedener Anbieter leichter und schneller entwickelt werden können (Robra-Bissantz und Lattemann 2017; Hein et al. 2019). Infolgedessen schließen sich heterogene Akteure (Menschen, Organisationen, etc.) in Service-Ökosystemen zusammen, in denen sie dann gemeinsam erstellte Dienstleistungen anbieten, wahrnehmen und individuelle oder gemeinschaftliche Mehrwerte generieren (Lusch und Nambisan 2015). Ein Service-Ökosystem ist dabei gemäß der SDL u. a. definiert durch den Wert, der auf Basis von Interaktionen für die Akteure entsteht. In diesem losen Netzwerk heterogener Akteure entstehen durch wiederholte Interaktionen im Laufe der Zeit erfolgreich gemeinsame Praktiken. Dies vereinfacht die Kollaboration und führt dazu, dass in einem ServiceÖkosystem Angebote erfolgreicher und effizienter co-kreiert werden und dem Kunden neue Werte angeboten werden können, die einzelne Dienstleister alleine nicht abbilden können (Lusch et al. 2010).

Digitalisierung bietet umfangreiche Möglichkeiten, Service-Ökosysteme mit intelligenten Anwendungen und Services für bzw. mit Kunden sowie Partnern umzusetzen (Pflaum und Fischer 2019). Die verschiedenen Stakeholder, welche am Service Ökosystem teilhaben, werden nachfolgend als Akteure bezeichnet und schließen Kunden und Partner ein. Verschiedene technikaffine Sektoren der Dienstleistungsbranche haben das Potenzial der Digitalisierung bereits in Form innovativer Services für sich erschlossen, wie z.B. der Finanzsektor, die Produktion oder die Logistik. Sogenannte personennahe Dienstleistungen $(P D)$, wie sie z. B. in der Hotel- und Gastronomiesektor, der Pflege, der Kinderbetreuung und dem Handwerk erbracht werden, stehen hier erst am Anfang (Kleinschmidt et al. 2016). Durch die direkten menschlichen Interaktionen spielte der Einsatz digitaler Technologien bisher eine untergeordnete Rolle innerhalb der PD (Robra-Bissantz et al. 2020). Dabei könnten jedoch insbesondere digitale Plattformen, die als passende Technologie zur Umsetzung der Service-Ökosysteme identifiziert wurden, zur Gestaltung von PD genutzt werden und so einen entscheidenden Wettbewerbsvorteil leisten (Barrett et al. 2015; Robra-Bissantz 2018). Während die technische Seite der Entwicklung von digitalen Plattformen schon eingehend betrachtet wurde (z.B. (de Reuver et al. 2018)), fehlen klare Richtlinien, die für die Gestaltung von kollaborativen Service-Plattformen herangezogen werden können (Fischer et al. 2020; Göbel und Cronholm 2016).

Diesen Bedarf erkennend, fördert das BMBF die Forschung und Entwicklung innovativer Lösungen für digitale, personennahe Dienstleistungen seit 2018 in insgesamt acht Verbundprojekten. Der nachfolgende Beitrag widmet sich der Betrachtung der in diesen Förderprojekten entstehenden Service-Ökosysteme. In der vorliegenden Fallstudie wird die sogenannte Service Canvas als Referenzrahmen herangezogen, 
mit deren Hilfe sich Dienstleistungsinnovationen rund um Personen gestalten lassen (Robra-Bissantz et al. 2020). Unter Anwendung der in diesem Referenzrahmen definierten Merkmale Individualisierung, Integration, Kollaboration und Digitale Services werden die Service-Ökosysteme hinsichtlich ihrer Umsetzung, Gestaltung und möglicher Potenziale analysiert. Hierbei beschreibt Individualisierung die Rolle der individuellen Akteurspräferenzen in Bezug auf Nachfrage, Nutzung und Bedeutung für die Entwicklung von Dienstleistungen, während es bei der Integration um die Art und Weise der Mitgestaltung geht. Auf die Bedeutung der Kollaboration und auch die Möglichkeiten, welche die digitalen Services (z.B. digitale Plattformen) dabei eröffnen, wurde bereits oben eingegangen. Anhand der Klassifizierung werden Gemeinsamkeiten abgeleitet, die wiederum für die Entwicklung zukünftiger Systeme herangezogen werden können.

\section{Theoretischer Hintergrund}

Für ein weiterführendes Verständnis werden in den folgenden Abschnitten die Aspekte Digitalisierung, Service-dominierte Logik (SDL), Service Ökosystem und Digitale Kundenschnittstellen erläutert und in Zusammenhang gestellt. Diese theoretische Auseinandersetzung dient als Grundlage, um die Beziehung zwischen der „Service Canvas“ und den Service-Ökosystemen nachvollziehen zu können.

\subsection{Digitalisierung}

Bei dem Begriff der Digitalisierung geht es generell immer um die Transformation des Analogen ins Digitale. Dieser, im Englischen als Digitization bezeichnete, rein technologische Vorgang ist die Grundlage einer richtungsgebenden Veränderung in Politik, Gesellschaft und Wirtschaft, die über das bloße digitale Abbilden von Prozessen und Informationen hinausgeht. Die weiterführende Interpretation, die so genannte Digitalization, beschreibt die Einführung digitaler Technologien in fast allen Lebens- und Arbeitsbereichen, die zu einem veränderten Miteinander und der Schaffung neuer Werte führt (Brennen und Kreiss 2016; Hess 2019). Der beachtliche gesellschaftliche Wandel durch die Verbreitung digitaler Technologien bietet vielfältige Chancen für Unternehmen, welche jedoch eine aktive Neuausrichtung auf digitale Märkte voraussetzen. Besonders die Relevanz digitaler Plattformen als Online-Geschäftsmodell, das Transaktionen zwischen mindestens zwei Akteuren ermöglicht, ist stark angestiegen und führt zunehmend zur Übernahme wichtiger Funktionen (wie z. B. Kommunikation, Zusammenarbeit, etc.) in der Wirtschaft und im Alltag. Für eine gelungene Digitalisierung von Geschäftsmodellen bedürfen besonders Unternehmen ohne bisherige digitale Strukturen Strategien mit klar definierten Handlungsfeldern. Schon seit einiger Zeit werden gleichbleibende und sich widerholende Aufgaben in Unternehmen im Rahmen einer (Teil-)Automatisierung mit IT vom Menschen auf den Computer übertragen (Hess 2019). Mittlerweile ermöglicht der Einsatz von Sensoren und Aktoren, neue Kommunikationsmöglichkeiten, die steigende Vernetzung und der Gebrauch von Enabler-Technologien auch die Automatisierung von komplexen Dienstleistungen. Darüber hinaus können neuarti- 
ge Anwendungen und Leistungen für die verschiedenen Akteure generiert werden (Schallmo 2016). Unternehmer sollten im Rahmen der Entwicklung von Dienstleistungsinnovationen den sinnvollen Grad der Digitalisierung individuell abwägen und entscheiden, in welchen Bereichen der Einsatz digitaler Technologien nützlich ist.

\subsection{Service-dominierte Logik (SDL)}

Mit den Veröffentlichungen der Service-dominierten Logik (SDL) (Vargo und Lusch 2004) existiert seit 2004 ein theoretisches Fundament, das die traditionelle Güterdominierte Sichtweise auf Wertschöpfung ablöst. Stammt diese Perspektive auch aus dem Bereich des Dienstleistungsmarketings, so stellt sie trotzdem die traditionelle Marketing-Perspektive auf den Kopf und gibt eine Erklärung für viele auf den Märkten bis dato beobachtbare Entwicklungen, denen es an theoretischer Begründbarkeit fehlte (Robra-Bissantz und Lattemann 2017). Traditionell aus der Industriegesellschaft heraus erwachsen, geht die Güter-dominierte Logik davon aus, dass Gütern in einer Wertschöpfungskette nach und nach Wert hinzugefügt wird. Der Wert für den Kunden entsteht durch seine Rolle als Käufer, durch den Besitz der Sache und über den Austausch von Gütern. Das produzierende Unternehmen schafft und kontrolliert den Wert; Dienstleistungen stehen eher am Ende der Wertschöpfungskette etwa in Form von Wartung. In der SDL rücken Produkte (Sach- und Dienstleistungen) in den Hintergrund und geben Raum für einen anderen Betrachtungsgegenstand: den Wert. Dieser wird über die Benutzung durch den Kunden und andere Akteure definiert, ist kontextabhängig (Value-in-Context) und individuell (Value-in-Use). Ein Produkt ist nur so gut, wie der Wert, den es dem einzelnen Akteuren bietet. Dabei spielt es für die Akteure keine Rolle, ob das Produkt eine Sach- oder Dienstleistung ist, solange sie zur Lösung seines Problems beiträgt. In einer Perspektive, in der Unternehmen Werte anbieten, aber nicht selbst schaffen können, verändern sich klassische Rollen und Machtbeziehungen von Kunden und Unternehmen: Der Kunde wird zunehmend aktiv in den Prozess der Leistungserbringung einbezogen und trägt zur Entstehung des Wertes bei (Vargo und Lusch 2004, 2008a).

\subsection{Service-Ökosysteme}

Das Service-Ökosystem, das sich auch aus der SDL ableitet, bietet Unternehmen eine ergänzende Perspektive in ihrer Weiterentwicklung. Definiert wird es als ,ein relativ eigenständiges, sich selbst anpassendes System von Akteuren, die Wert auf die Integration von Ressourcen legen, und die durch gemeinsame institutionelle Vereinbarungen und gegenseitige Wertschöpfung durch den Austausch von Dienstleistungen verbunden sind" (Vargo und Lusch 2016, S. 16). Die Anwendung der Service-Ökosystem-Perspektive eignet sich in der Entwicklung von Dienstleistungen als strukturiertes, dynamisches Instrument zur Untersuchung von Interaktionen und Austauschhandlungen zwischen verschiedenen Stakeholdern. In dieser Betrachtung sind alle Akteure und Technologien mögliche Bereitsteller von tangiblen und intangiblen Ressourcen für die (gemeinsame) Erstellung und Realisierung von Wertangeboten (also Dienstleistungen) (Lusch und Nambisan 2015). Dieser Perspektivwechsel führt zu einer Veränderung des Begriffs des Wertes, da die Value Creation 
durch gemeinsame Anstrengungen von Akteuren (z. B. Lieferanten, Dienstleistungsanbieter, Technologien) zur Value Co-Creation wird (Vargo et al. 2008). In diesem Konzept wird der Value-in-Use durch die Anwendung von Ressourcen zur Realisierung eines Wertes von einen oder mehreren Akteuren bestimmt, der sich je nach Kontext unterscheidet.

Die steigende Bedeutung der Service-Ökosystem-Perspektive spiegelt sich im Erfolg von digitalen Plattformen wider, die heterogenen, global vernetzten Akteuren ermöglichen, Dienstleistungen kollaborativ zu entwickeln und anzubieten. Die Akteure können durch digitale Plattformen leichter im Ökosystem vorhandene Ressourcen identifizieren, anbieten und nachfragen. Hierdurch wird deutlich, wie Technologien digitalisierte Ökosystem ermöglichen und wie menschliche Akteure ihr Verhalten anpassen können (Sklyar et al. 2019). Die Service-Ökosystem-Perspektive fördert so ein besseres Verständnis über das Zusammenspiel verschiedener Akteure, das auf die gemeinsame Realisierung des Value-in-Use fokussiert (Vargo und Lusch 2008a; Merz et al. 2009).

\subsection{Digitale Schnittstellen}

Digitalisierung in Service-Ökosystemen ermöglicht die Umsetzung von Digitalen Schnittstellen, die dazu beitragen, Innovationen in Akteursbeziehungen und ServiceEntwicklungsaktivitäten zu fördern (Um und Yoo 2016). Um IT-fähige Dienstleistungen zu entwickeln und einen Wettbewerbsvorteil im Hinblick auf die kombinierte Nutzung von Technologien zu erzielen, suchen Unternehmen nach anderen Unternehmen, mit denen sie zusammenarbeiten und Allianzen bilden können (Barrett et al. 2012). Eine Herausforderung von Service-Ökosystemen liegt im Aufbau solcher Strukturen, die einerseits Partizipation ermöglichen und gleichzeitig erlauben die Koordination von Interaktionen zwischen Netzwerkakteuren hinsichtlich ihrer Art und Weise zu prägen (Lusch und Nambisan 2015). Wichtig ist es, Schnittstellen bereitzustellen und den Zugriff auf geeignete Ressourcen oder Ressourcenbündel zu erweitern, um den kollaborativen Innovationsprozess zu unterstützen und die Möglichkeiten für Service-Innovationen zu verbessern (Lusch und Nambisan 2015). Vor diesem Hintergrund haben sich Serviceangebote von Single-Interface-Systemen zu Multi-Interface-Systemen entwickelt, bei dem die Technologie eine zentrale Rolle sowohl für Interaktionen im Vordergrund als auch für Unterstützungsprozesse im Hintergrund einnimmt (Patrício et al. 2008). Während Unternehmen beim Design von Dienstleistungen früher hauptsächlich die Bereitstellung physischer Schnittstellen, wie z.B. Geschäfte, beachten mussten, erfordern moderne Serviceangebote einen digital unterstützten Service-Schnittstellen-Mix. Der richtige Einsatz neuer Technologien ermöglicht es, Akteursinteraktionen und im Hintergrund laufende Supportprozesse zu koordinieren und dabei Kompetenzen und Ressourcen zu definieren, die für ein zufriedenstellendes Dienstleistungserlebnis erforderlich sind (Patrício et al. 2008). 


\section{Vorgehen und Fallbeispiele}

Die Service Canvas ist ein Teilergebnis des vom BMBF Begleitforschungsprojektes BeDien, welches seit 2018 in der Bekanntmachung „Personennahe Dienstleistungen“ gefördert wird (siehe. Robra-Bissantz et al. 2020). Im Rahmen der Innovationsentwicklung von PD dient die Service Canvas (siehe Tab. 1) dazu, wesentliche Bereiche zur Gestaltung aufzuzeigen und ihnen Betrachtungspunkte im Entwicklungsprozess gegenüberzustellen. Einer der drei Gestaltungsbereiche ist das Service-Ökosystem, dem zur Entwicklung die Betrachtungspunkte Individualisierung, Integration, Kollaboration und Digitales Service Design zugeordnet werden. In Tab. 1 werden alle Gestaltungsbereiche und Entwicklungslinien sowie exemplarische Ausprägungen deren Kombination dargestellt.

Um die Service Canvas im Bereich der Service-Ökosysteme auf ihre Gültigkeit zu testen, wurde eine Fallstudie durchgeführt. Hierbei wurden die Service-Ökosysteme der acht in der gleichen Bekanntmachung geförderten Verbundprojekte exemplarisch beschrieben. In gemeinsamen Workshops mit den Projekten und durch Interviews mit Projektbeteiligten wurden Kernanforderungen, Ziele und Lösungswege hinsichtlich der Gestaltung der Service-Ökosysteme in personennahen Dienstleistungen herausgearbeitet. Für die nachfolgende Darstellung der Ergebnisse wurden pro Betrachtungspunkt des Entwicklungsprozesses jeweils zwei am besten geeignete Projektbeispiele ausgewählt. Diese Auswahl wird in Tab. 2 zusammengefasst:

Tab. 1 Service Canvas und exemplarische Ausprägungen. (Robra-Bissantz et al. (2020))

\begin{tabular}{|c|c|c|c|c|c|}
\hline & & \multicolumn{4}{|l|}{ Entwicklungslinien } \\
\hline & & Individualisierung & Integration & Kollaboration & Digitale Services \\
\hline \multirow[t]{3}{*}{$\begin{array}{l}\text { Gestaltungs- } \\
\text { bereiche }\end{array}$} & Value-in-Use & $\begin{array}{l}\text { Individuelle, situ- } \\
\text { ierte Angebote }\end{array}$ & $\begin{array}{l}\text { Kundenkom- } \\
\text { petenzen }\end{array}$ & $\begin{array}{l}\text { Gemeinsame } \\
\text { Dienstleis- } \\
\text { tungsentwick- } \\
\text { lung }\end{array}$ & $\begin{array}{l}\text { Digitale Dienst- } \\
\text { leistungen }\end{array}$ \\
\hline & $\begin{array}{l}\text { Value-in- } \\
\text { Interaction }\end{array}$ & $\begin{array}{l}\text { Individuelle Pas- } \\
\text { sung }\end{array}$ & Kundenwissen & $\begin{array}{l}\text { Soziale Inter- } \\
\text { aktionen }\end{array}$ & $\begin{array}{l}\text { User-Experience } \\
\text { Design }\end{array}$ \\
\hline & $\begin{array}{l}\text { Service- } \\
\text { Ökosystem }\end{array}$ & $\begin{array}{l}\text { Dynamische An- } \\
\text { passung eigener } \\
\text { Kompetenzen }\end{array}$ & $\begin{array}{l}\text { Wertkompe- } \\
\text { tenz-Planung }\end{array}$ & $\begin{array}{l}\text { Agilität durch } \\
\text { Kollaboration }\end{array}$ & $\begin{array}{l}\text { Digitale Kun- } \\
\text { denschnittstellen }\end{array}$ \\
\hline
\end{tabular}

Tab. 2 Einordnung der Fallbeispiele in die Service Canvas

\begin{tabular}{|c|c|c|c|c|c|}
\hline & & \multicolumn{4}{|c|}{ Entwicklungslinien } \\
\hline & & $\begin{array}{l}\text { Individuali- } \\
\text { sierung }\end{array}$ & Integration & Kollaboration & $\begin{array}{l}\text { Digitale } \\
\text { Services }\end{array}$ \\
\hline \multirow{3}{*}{$\begin{array}{l}\text { Gestaltungs- } \\
\text { bereiche }\end{array}$} & Value-in-Use & $\otimes$ & $\otimes$ & $\otimes$ & $\nabla$ \\
\hline & Value-in-Interaction & $\otimes$ & $\otimes$ & $\otimes$ & $\otimes$ \\
\hline & Service-Ökosystem & $\begin{array}{l}\text { MYOW } \\
\text { ARBAY }\end{array}$ & $\begin{array}{c}\text { Athene } 4.0 \\
\text { proDruck }\end{array}$ & $\begin{array}{l}\text { INSELpro } \\
\text { LivingSmart }\end{array}$ & $\begin{array}{c}\text { KUSTOMA } \\
\text { LidA }\end{array}$ \\
\hline
\end{tabular}




\subsection{Beispiel MYOW und ARBAY - Individualisierung im Service-Ökosystem}

Bedingt durch den Trend einer zunehmenden Individualisierung von Produkten und Dienstleistungen ist innerhalb der vergangenen Jahre die Nachfrage nach bedarfsund nutzergerecht erbrachten Dienstleistungen immens gestiegen. Dem Kunden wird eine individuell angepasste Dienstleistung oder ein Produkt zu einem unwesentlich höheren Preis gegenüber Massenprodukten geboten; aus Unternehmenssicht führen individualisierte, stark nach Kundenpräferenz ausgerichtete Angebote zu Umsatzsteigerungen. Dabei wird der Wert von Dienstleistungen nahezu immer individuell determiniert (Vargo und Lusch 2008b; Grönroos 2011), sodass Dienstleistungsgeber und -nehmer eng in einem interaktiven Prozess kooperieren müssen. Die Projekte ARBAY und MYOW zeigen auf verschiedene Art und Weisen auf, welch bedeutende Rolle die Individualisierung im Entwicklungsprozess der eigenen Leistung einnehmen kann.

Das Projekt „Augmented-Reality basierte interaktive Beratungs-, kooperative Konfigurations- und Verkaufsplattform für hochvariante und individualisierbare Güter" (ARBAY) hat sich etwa zum Ziel gesetzt, die Beratung und Konfiguration von großen, hochindividualisierbaren Gütern wie z.B. Möbeln ins heimatliche Wohnzimmer zu bringen. Um bereits vor Lieferung beurteilen zu können, wie die Produkte in den eigenen vier Wänden wirken, entwickelt und erprobt das Forschungsprojekt eine digitale Augmented-Reality- (AR-)Verkaufsplattform. Über diese Plattform soll der Kunde durch den Einsatz einer AR-Brille das Möbelstück im eigenen Heim konfigurieren und vorab sehen können. Ein automatisierter Beratungsassistent in Form eines Chatbots soll dem Kunden dabei unterstützend zur Seite stehen, um eine erste Vorauswahl und Begutachtung des Produkts vorzunehmen. Reicht der virtuelle Berater nicht aus, bietet die Plattform bei Bedarf eine persönliche Beratung mit einem Fachmann durch Liveschaltung an. Anschließend kann mit einem Verkäufer eine Detailabsprache durchgeführt werden. Der in diesem Kontext erwähnte Einsatz von AR-Technologien sowie des automatisierten Chatbots schafft nicht nur neue digitale Vertriebswege, sondern stellt beispielhaft auch Möglichkeiten dar, wie digitale Services zur Individualisierung hochvarianter Produkte beitragen kann.

Getreu dem Motto „Make Your Own Wearables“ nimmt sich das gleichnamige Forschungsprojekt (MYOW) des Problems fehlender digitaler Austauschplattformen für selbstgefertigte Produkte in der Bekleidungsbranche an. Dank moderner Technologie ist es bereits möglich, Bekleidung mit intelligenten Sensoren auszustatten und den Textilien somit zusätzlich nützliche Funktionalitäten zu verleihen. Bedingt durch den Trend des Do-it-Yourself (DIY) gewinnt handgemachte Kleidung auch in der Gesellschaft immer mehr an Bedeutung. Die Herstellung intelligenter Kleidung erfordert jedoch erweiterte Kenntnisse in den Bereichen der Programmierung und Elektrotechnik. Fehlt der Zugang zu einer professionellen ProduktionsInfrastruktur als auch die Expertise, diese einzusetzen, wird es schwierig Kleidungsstücke an die Qualität professioneller Produkte anzuschließen und diese individuell anzupassen. Obwohl bereits verschiedene Portale Vertriebsmöglichkeiten für selbstgefertigte Produkte zur Verfügung stellen, existiert kein systematischer Austausch zwischen Akteuren oder etwa die Möglichkeit fehlende Kompetenzen hinzuzuziehen. An dieser Stelle kommen die Forschungsarbeiten aus MYOW zum Einsatz: 
Die Entwicklung digitaler Dienstleistungsinfrastrukturen soll Herstellern das Anfertigen professioneller individualisierter Produkte, den Dienstleistern den Ausbau der bedarfsorientierten eigenen Angebote, sowie allen Anbietern die Bildung von Serviceketten ermöglichen. Ähnlich wie bei dem zuvor erwähnten Forschungsprojekt ARBAY muss auch hier in einem interaktiven Prozess kooperiert werden, damit auf den digital angereicherten Service-Spaces sowohl DIY-Maker, Designer, Dienstleister und Hersteller mit dem Ziel professionelle individualisierte Produkte anfertigen zu können, systematisch miteinander arbeiten.

\subsection{Beispiel Athene 4.0 und proDruck - Integration im Service-Ökosystem}

In Handwerksbetrieben sind die meisten internen Prozesse noch vollständig ana$\log$, weswegen es für die dazugehörigen Unternehmen äußerst wichtig ist, neue Geschäftsmodelle mit digitalisierten Prozessen einzurichten, um eine höhere Effizienz der Akteursinteraktionen zu erreichen. In diesem Zusammenhang versucht das Projekt „Personenzentrierte Digitalisierung zur Zukunftssicherung des Handwerks“ (Athene 4.0) unter Verwendung des „Digital Coach“-Ansatzes ein Dienstleistungskonzept für die Bereitstellung von Handwerksdienstleistungen über eine digitale Serviceplattform zu entwickeln. Athene 4.0 vernetzt Kunden und Handwerksbetriebe unter- und miteinander und bietet leicht zugängliche Lösungen zur Digitalisierung interner Prozesse des beruflichen Alltags im Handwerk. Durch die vereinfachte Bereitstellung und die kontinuierliche Ergänzung von Information seitens aller Akteure (Handwerksbetriebe und Kunden), sowie die Einrichtung von kürzeren Kommunikationswegen auf der digitalen Plattform, können Arbeitsprozesse effizienter und kundenorientierter gestaltet werden. So bietet Athene 4.0 z. B. eine digitale Datenbank mit aktuellen Informationen zu Handwerksbetrieben, die es dem Kunden ermöglicht, ohne langwieriges Suchen den richtigen Handwerker in ihrer Nähe zu finden, technische Fragen zu klären und sich beraten zu lassen, wobei Kundenanfragen sogar Gewerke-übergreifend koordiniert werden können. Diese Integration von Wissen und Kommunikation ohne physische, zeitliche oder geographische Einschränkungen mithilfe einer anwendungsorientierten digitalen Plattform, führt zu und begleitet die Akteure bei Prozessinnovationen. Die dadurch verkürzten Kommunikationswege, die verbesserte Kundenanpassung von Dienstleistungen und die optimierte Informationsabfrage stellt einen erheblichen Wettbewerbsvorteil für die teilnehmenden Handwerksbetriebe dar.

Auch im Forschungsprojekt „3D-Druck - Inklusion von Menschen mit Behinderungen in die Arbeitswelt" (proDruck) steht die Integration verschiedener Akteure im Vordergrund des zugrundeliegenden Service-Ökosystems. Ziel von proDruck ist die Entwicklung eines ganzheitlichen Beschäftigungsmodelles, das auf einer OnlinePlattform basierend 3D-Baugruppen für Menschen mit Behinderungen anbietet, die durch die Verwendung von Technologien wie 3D-Druck entstehen. Ein wichtiges Merkmal dieser Dienstleistung ist, dass Kunden als Ko-Produzenten integriert werden. Ko-Produktion ist definiert als ein Prozess der kundenindividuellen Massenproduktion, bei dem Kunden aktiv und iterativ an der Lösungsentwicklung und der Serviceentwicklung teilnehmen (Morelli et al. 2009). Kunden von proDruck werden also als aktive Akteure eingebunden, um die Serviceentwicklung zu verbessern 
indem sie z.B. mit einer Vielzahl von Experten bezüglich der Anwendungsmöglichkeiten der verschiedenen Produkte interagieren und mit anderen Akteuren ihre Erfahrungen austauschen. Sie haben darüber hinaus die Möglichkeit, selbst gestaltete Komponenten anderen Anwendern zur individuellen Weiterentwicklung und zum Kauf zur Verfügung zu stellen und sich mithilfe der Q\&A-Funktion mit anderen Akteuren (d.h. proDruck, Hersteller von 3D-Baugruppen) auszutauschen und zusammenzuarbeiten. Durch die Integration von heterogenen Akteuren mithilfe dieser digitalen Plattform, ermöglicht proDruck die kollaborative Entwicklung und Distribution von erschwinglichen Baugruppen, die an die individuellen Anforderungen der Kunden angepasst sind. Hierdurch wird nicht nur die Qualität, sondern auch das Dienstleistungserlebnis und die Nachfrage nach besser personalisierten Produkten verbessert.

\subsection{Beispiel INSELpro und LivingSmart - Kollaboration im Service- Ökosystem}

Das Projekt „Intelligentes Servicesystem für lokal vernetzte Prosumenten“ (INSELpro) liefert mit der Entwicklung einer Dienstleistungsplattform unter dem Ansatz „Nachbarschaftshilfe 2.0“ ein Beispiel für Kollaboration in einem Service-Ökosystem. Innerhalb des Projektes entwickelt das Konsortium ein Dienstleistungskonzept für die Erbringung von Nachbarschaftsdiensten. Das Konzept der Nachbarschaftshilfe findet insbesondere in eher ländlichen Regionen großen Anklang, in urbanen Gebieten hingegen kennt man seine Nachbarn weniger oder häufig gar nicht. Aufgrund ihrer Lebenssituation sind Menschen jedoch auf Hilfe angewiesen, sei es im Krankheitsfall oder bei der Kinderbetreuung. INSEL.pro möchte dieses Potenzial der gegenseitigen unentgeltlichen Nachbarschaftshilfe daher auch in urbanen Gebieten nutzen, sodass die individuellen Fähigkeiten der einzelnen Bewohner im Mittelpunkt stehen und diese sich über die Dienstleistungsplattform gegenseitig unterstützen können. Besonderer Wert wird dabei auf die Gestaltung und Umsetzung von Motivationsstrategien gelegt, um eine langfristige Nutzung der Plattform zu fördern und die Zusammenarbeit anzuregen. Die Plattform ist so gestaltet, dass Gesuche und Angebote intuitiv gefunden werden können und eine Auswahl nach Interessen möglich ist. Die initiale Registrierung auf der Plattform erfolgt dabei immer durch den/die sogenannten QuartiersmanagerIn - eine menschliche Kontaktperson vor Ort. Die Überprüfung der persönlichen Daten des zu registrierenden Nutzers senkt die Hemmschwelle der Nutzung, denn ohne ein tiefergehendes Vertrauen in die Authentizität der Profile wäre zum Beispiel eine nachbarschaftliche Kinderbetreuung kaum denkbar. Dieses Vertrauen bezieht sich auch darauf, dass eine Dienstleistung nicht nur einseitigen Nutzen bringt, sondern ein gegenseitiger Austausch stattfindet. Durch den Einsatz von z.B. Gamification wird schon bei der Gestaltung darauf geachtet, dass engagierte Akteure motiviert bleiben. Da die Bewohner sowie die gemeinnützigen Partner zu Prosumenten werden, d.h. Dienstleistungsgeber und -nehmer zugleich, müssen alle beteiligten Akteure in einem interaktiven Prozess miteinander kollaborieren.

Im Projekt „Web-basierte Plattform für Buchungen von wohnungsnahen Dienstleistungen in Quartierten“ (LivingSmart) geht es vorrangig um die Einbindung pro- 
fessioneller wohnungsbezogener Dienstleistungen. LivingSmart bietet Mietern eine Plattform, auf der sämtliche Dienstleistungen rund ums Wohnen zu finden sind, ergänzt um die Bereiche Mobilität, Betreuung, Ernährung und Empowerment. Durch intelligente Algorithmen sollen dabei die speziellen Bedarfe der (in diesem Kontext privaten) Nutzer berücksichtigt werden. LivingSmart nutzt somit die Daten der Mieter, um zielgerichtete Angebote entwickeln und bereitstellen zu können. Auch hier ist Vertrauen für die Kollaboration entscheidend: Eine Weitergabe von Daten erfolgt nur, wenn der Nutzer über die Verwendung ausreichend informiert wurde. Unter diesen Bedingungen lassen sich dann neue Werte für die beteiligten Akteure kreieren, von denen alle Akteure profitieren können. Durch ein Feedback-System wird gewährleistet, dass Ideen für neue Dienste und für Verbesserungen direkt von den Nutzern eingereicht werden können, wodurch die Loyalität in der Nutzung steigen kann. Zusätzlich bietet LivingSmart über eine Art Forum die Möglichkeit zur Zusammenarbeit zwischen den Nutzern. Diese können hierüber Dienstleistungen und Gegenstände tauschen oder verleihen, wodurch die Verbundenheit untereinander innerhalb des Service-Ökosystems gestärkt wird. Je öfter und je positiver diese Zusammenarbeit stattfindet, desto schneller baut sich Vertrauen auf. Das Angebot an unterschiedlichen Dienstleistungsbündeln erfordert ein sehr hohes Maß an Kollaboration. So wird im Bereich der Betreuung etwa ein Notfallbetreuungsnetz mit sowohl professionellen als auch mit ehrenamtlichen Teilnehmern aufgebaut - für alle hier involvierten Akteure ist eine effektive Zusammenarbeit essentiell, damit die Dienstleistungserbringung problemlos abgewickelt werden kann. Dies zeigt wie wichtig die Beachtung und Ausgestaltung von Kollaboration für ein Unternehmen sein kann. Im besten Fall kann dabei ein gemeinsames Wertesystem aufgebaut werden, welches die Bindung an das Service-Ökosystem stützt und es langfristig sichert.

\subsection{Beispiel KUSTOMA und LidA - Digitale Services im Service Ökosystem}

In den letzten Jahrzehnten hat sich die Beschäftigungssituation in Deutschland grundsätzlich verändert. Dem steigenden Anteil von Doppelverdiener-Haushalten rücken Angebote wie Ganztagesbetreuung nach. Diese Anpassung erfolgt längst nachdem der Bedarf entsteht. Aktuelle Entwicklungen hin zu Gleitzeit- und HomeOffice-Modellen verbreiten sich durch die hohe Konkurrenz um Fachkräfte schnell in deutschen Betrieben. Welche Antworten sich mit der Zeit durch staatliche Angebote entwickeln werden, bleibt abzuwarten. In diesem Kontext entwickelt das Forschungsprojekt ,Kinderbetreuung unterstützt durch online implementierte und mobile Applikationen" (KUSTOMA) einen ganzheitlichen, flexiblen Ansatz zur Entlastung von ArbeitnehmerInnen, der diese unterstützt den privaten und professionellen Alltag zu vereinen. Dienstleistungen wie Kinderbetreuung und andere haushaltsnahe Angebote werden über eine digitale Plattform angeboten, welche alle Prozessschritte von der Vermittlung bis zu Vergütung digital unterstützen soll, um die Auftragnehmer effektiv zu entlasten. Eine Schlüsselherausforderung der Plattform ist, zwischen Leistungsanbietern und -nachfragern ein Vertrauensverhältnis zu fördern, das es erlaubt größtenteils fremde Personen in das intime Umfeld der eigenen vier Wände einzuladen und darüber hinaus die Betreuung der eigenen Kinder oder Tiere zu überlassen. 
Effiziente und wertstiftende Prozesse sollen Akteure langfristig auf der Plattform halten, obwohl sich bereits ein Vertrauensverhältnis von Anbietenden und Nachfragenden eingestellt hat. Die Vereinfachung des Buchungsprozesses gilt es daher im Verlauf der Wiederinanspruchnahme tendenziell so zu steigern, dass die Akteure incentiviert werden langfristig und lückenlos die Plattform zu nutzen, ohne so Barrieren zu schaffen, welche den Eintritt der Akteure hemmen. Die vollumfängliche Digitalisierung des Prozesses bietet in diesem Kontext diverse Möglichkeiten, den Akteuren die Zusammenarbeit zu erleichtern, ob nun bei der Zahlung, Versteuerung, Versicherung und Organisation sowohl auf Anbieter- als auch auf Nachfrageseite. Der digitale Service der Plattform wird so zum Kern der Interaktion zwischen den Akteuren des Service-Ökosystems und erzeugt neben individuellen Vorteilen über steigende Angebote und Nutzer zunehmende Reichweite und Auswahl für die Akteure. Diese zentralen Leistungen werden durch einen Intermediär angeboten, der auch weitere individuelle Anfragen, Hilfegesuche und Angebote auf der Plattform durch effiziente digitale Services verwaltet und einbindet, wodurch er nachhaltig die Innovationskraft sichert und Legitimität schafft.

Digitalisierte Dienstleistungen können in einem hoch persönlichen Kontext, wie haushaltsnahen Dienstleistungen, aber auch in hoch professionellen Umfeldern wirksam eingesetzt werden, indem durch nutzerzentrierte Funktionen individuelle Leistungen erbracht und Werte geschaffen werden. Das Forschungsprojekt „Lernen in der digitalisierten Arbeitswelt" (LidA) hat es sich zur Aufgabe gemacht, den digitalen Wandel in Unternehmen durch eben einen solchen individualisierten digitalen Service zu unterstützen. Die Lehr- und Lernplattform ermittelt zu diesem Zweck mitarbeiterspezifische Kompetenzbedarfe, die in individuelle Lernentwicklungspfade übersetzt werden. Auf Basis einer Open-Source-Plattform sollen Lernmodule barrierefrei zur Verfügung gestellt und proaktiv von Beschäftigten genutzt werden. Der Lernfortschritt wird analysiert und in Fortschrittsstufen dargestellt, um dem Nutzer stetig Feedback zur Selbsteinschätzung zu vermitteln.

Durch diese individualisierten Weiterbildungsmaßnahmen wird eine zentrale Herausforderung der Digitalisierung adressiert, die sich in den stetig neuen Anforderungen begründet. Bei der Vielzahl der Veränderungen ist die kontinuierliche Weiterbildung ein Kernelement für die langfristige Sicherung der Wettbewerbsfähigkeit. Die Konzeption von zeitgemäßen und individualisierten Weiterbildungsmaßnahmen ist in diesem Rahmen eine enorme Herausforderung für Unternehmen, die Kompetenzbedarfe von hunderten oder tausenden Mitarbeitern kennen und bewerten müssen. Durch Skalierungs- und Individualisierungspotenziale von zeitgemäßen digitalen Services, die durch die verschiedenen Akteure in Ökosystemen gepflegt und erweitert werden können, bietet eine digitale Lehr- und Lernplattform eine adäquate digitalisierte Lösung um Beschäftigte durchs Wissensbildende und -verbreitende Maßnahmen zu unterstützen.

\section{Fazit}

Dargestellt wurde, wie Service-Ökosysteme aktuelle Trends der Dienstleistungsentwicklung zusammenführen und es ermöglichen, in einem Akteursnetzwerk mit 
Kunden gemeinsam innovative Wertangebote zu schaffen. Anhand der vorgestellten Fallbeispiele wurde verdeutlicht, dass digitale Technologien Service-Ökosysteme zu einem attraktiven Instrument ergänzen: Augmented Reality und Chatbots ( $A R B A Y)$, 3D-Drucker (proDruck) oder Wearables $(M Y O W)$ sind hier als Beispiele zu nennen. Unternehmen aus dem Bereich der personennahen Dienstleistungen können dieses Instrument zur Aktivierung des eigenen Digitalisierungspotenzials heranziehen. Sowohl in der Rolle von Plattformbetreibern, wie am Beispiel LivingSmart und LidA, als auch in der Rolle von Komplementoren, wie die Handwerksbetriebe bei Athene 4.0, die kinderbetreuenden Einrichtungen von KUSTOMA oder die privaten Akteure bei INSELpro, können Anbieter von PD Service- und Prozessinnovationen generieren. Die Service Canvas des Begleitforschungsprojektes BeDien bietet dabei einen Referenzrahmen, um zwischen Service-Ökosystemen völlig unterschiedlicher Bereiche einen Bezug herzustellen. So auch geschehen für die acht Forschungsprojekte der dazugehörigen Förderung, deren thematische Schwerpunkte äußerst heterogen gewählt sind. Im Rahmen des Begleitforschungsprojektes BeDien verfolgen wir einen gestaltungsorientierten Forschungsansatz um generalisierbares, praktisch relevantes Wissen für die Konzeptionierung und Evaluation von fortschrittlichen, PD zu generieren. Der durch die Canvas geschaffene Bezug kann sowohl zur Gestaltung als auch zur Analyse verwendet werden und erlaubt die Betrachtung von Merkmalen wie Individualisierung, Integration, Kollaboration und Digitale Services. Dadurch wird es möglich, Stärken, Schwerpunkte und Potenziale von Service-Ökosystemen zu identifizieren und für das eigene Angebot zu lernen, wie Digitalisierung als Grundlage für die Zusammenarbeit zielgerichtet eingesetzt werden kann.

Förderung Dieser Beitrag ist Teil des vom Bundesministerium für Bildung und Forschung geförderten Verbundprojekts „BeDien - Begleitforschung Personennahe Dienstleistungen“ (Förderkennzeichen: 02K17A080-81).

Funding Open Access funding provided by Projekt DEAL.

Open Access Dieser Artikel wird unter der Creative Commons Namensnennung 4.0 International Lizenz veröffentlicht, welche die Nutzung, Vervielfältigung, Bearbeitung, Verbreitung und Wiedergabe in jeglichem Medium und Format erlaubt, sofern Sie den/die ursprünglichen Autor(en) und die Quelle ordnungsgemäß nennen, einen Link zur Creative Commons Lizenz beifügen und angeben, ob Änderungen vorgenommen wurden.

Die in diesem Artikel enthaltenen Bilder und sonstiges Drittmaterial unterliegen ebenfalls der genannten Creative Commons Lizenz, sofern sich aus der Abbildungslegende nichts anderes ergibt. Sofern das betreffende Material nicht unter der genannten Creative Commons Lizenz steht und die betreffende Handlung nicht nach gesetzlichen Vorschriften erlaubt ist, ist für die oben aufgeführten Weiterverwendungen des Materials die Einwilligung des jeweiligen Rechteinhabers einzuholen.

Weitere Details zur Lizenz entnehmen Sie bitte der Lizenzinformation auf http://creativecommons.org/ licenses/by/4.0/deed.de. 


\section{Literatur}

Barrett M, Davidson E, Prabhu J, Vargo SL (2015) Service innovation in the digital age: key contributions and future directions. MIS Q 39:135-154

Barrett MI, Davidson EJ, Fayard A-L, Vargo SL, Yoo Y (2012) Being innovative about service innovation: service, design and digitalization. In: 33rd Proceedings of the International Conference of Information Systems (ICIS)

Brennen JS, Kreiss D (2016) Digitalization. In: Jensen KB, Rothenbuhler EW, Pooley JD, Craig RT (Hrsg) The international encyclopedia of communication theory and philosophy, S 1-11

Fischer S, Lohrenz L, Lattemann C, Robra-Bissantz S (2020) Critical design factors for digital service platforms: a literature review. In: Proceedings of the European Conference of Information Systems (ECIS)

Göbel H, Cronholm S (2016) Illustrating emerging design principles enhancing digital service platforms. In: Proceedings of the 11th International Conference on Design Science Research in Information Systems and Technology (DESRIST)

Grönroos C (2011) A service perspective on business relationships: the value creation, interaction and marketing interface. Ind Mark Manag 40:240-247

Hein A, Schreieck M, Riasanow T et al (2019) Digital platform ecosystems. Electron Markets 1:1-12

Hess T (2019) Digitale Transformation strategisch steuern: Vom Zufallstreffer zum systematischen Vorgehen. Springer, Wiesbaden

Kleinschmidt S, Burkhard B, Hess M, Peters C, Leimeister JM (2016) Towards design principles for aligning human-centered service systems and corresponding business models. In: Proceedings of the 37th International Conference of Information Systems (ICIS)

Lusch RF, Nambisan S (2015) Service innovation: a service-dominant logic perspective. MIS Q Manag Inf Syst 39:155-175

Lusch RF, Vargo SL, Tanniru M (2010) Service, value networks and learning. J Acad Mark Sci 38:19-31

Merz MA, He Y, Vargo SL (2009) The evolving brand logic: a service-dominant logic perspective. J Acad Mark Sci 37:328-344

Morelli N, Roy R, Shehab E, Tiwari A (2009) Service as value co-production: regraming the service design process. J Manuf Technol Manag 20:568-590

Patrício L, Fisk RP, Falcão e Cunha J (2008) Designing multi-interface service experiences: the service experience blueprint. J Serv Res 10:318-334

Pflaum A, Fischer C (2019) Von der Pipeline zur Plattform. In: Ulrich P, Baltzer B (Hrsg) Wertschöpfung in der Betriebswirtschaftslehre: Festschrift für Prof. Dr. habil. Wolfgang Becker zum 65. Geburtstag. Springer, Wiesbaden, S 409-429

de Reuver M, Sørensen C, Basole RC (2018) The digital platform: a research agenda. J Inf Technol 33:124-135

Robra-Bissantz S (2018) Entwicklung von innovativen Services in der Digitalen Transformation. In: Bruhn M, Hadwich K (Hrsg) Service business development. Springer, Wiesbaden, S 261-288

Robra-Bissantz S, Lattemann C (2017) 7 rules of attraction. HMD 54:639-658

Robra-Bissantz S, Latteman C, Guerrero R, Lux AM, Redlich B, Fischer S (2020) Der Mensch als Teil der Innovatin - eine „Service Canvas“ als anwendungs-orientierter Bezugsrahmen. In: Bruhn, Hadwich (Hrsg) Automatisierung und Personalisierung von Dienstleistungen. Springer Gabler, Wiesbaden

Schallmo DRA (2016) Jetzt digital transformieren: So gelingt die erfolgreiche Digitale Transformation Ihres Geschäftsmodells. Springer, Wiesbaden

Sklyar A, Kowalkowski C, Tronvoll B, Sörhammar D (2019) Organizing for digital servitization: a service ecosystem perspective. J Bus Res 104:450-460

Um S, Yoo Y (2016) The co-evolution of digital ecosystems completed research paper. In: Proceedings of the 37th International Conference on Information Systems (ICIS)

Vargo SL, Lusch RF (2004) Evolving to a new dominant logic for marketing. J Mark 68:1-17

Vargo SL, Lusch RF (2008a) Service-dominant logic: continuing the evolution. J Acad Mark Sci 36:1-10

Vargo SL, Lusch RF (2008b) From goods to service(s): divergences and convergences of logics. Ind Mark Manag 37:254-259

Vargo SL, Lusch RF (2016) Institutions and axioms: an extension and update of service-dominant logic. J Acad Mark Sci 44:5-23

Vargo SL, Maglio PP, Akaka MA (2008) On value and value co-creation: a service systems and service logic perspective. Eur Manag J 26:145-152 